\title{
Power quality metrics for DC grids with pulsed power loads
}

\author{
Andrea Mariscotti ${ }^{1}$ \\ ${ }^{1}$ DITEN, University of Genova, Via Opera Pia 11A, 16145 Genova, Italy
}

\section{ABSTRACT}

DC grids can effectively feed pulsed power loads (PPLs), integrating local energy storage to minimize the impact on other connected loads and implementing buffered sub-grids to isolate susceptible loads even more. The identification of regulatory limits for PPL integration and DC grid response and the assessment of PPL impact necessitate suitable Power Quality (PQ) metrics. Existing indexes and limits (e.g. ripple and distortion, voltage fluctuation) are compared to other metrics, derived from control methods and knowledge of the peculiar characteristics of PPL and interaction with DC grids in some examples. The objective is a unified approach for PQ metrics suitable for a wide range of DC grids and in particular to the behavior of PPLs.

\section{Section: RESEARCH PAPER}

Keywords: DC grid; power quality; pulsed power load

Citation: Andrea Mariscotti, Power quality metrics for DC grids with pulsed power loads, Acta IMEKO, vol. 10, no. 2, article 22, June 2021, identifier: IMEKOACTA-10 (2021)-02-22

Section Editor: Giuseppe Caravello, Università degli Studi di Palermo, Italy

Received February 17, 2021; In final form April 24, 2021; Published June 2021

Copyright: This is an open-access article distributed under the terms of the Creative Commons Attribution 3.0 License, which permits unrestricted use, distribution, and reproduction in any medium, provided the original author and source are credited.

Corresponding author: Andrea Mariscotti, e-mail: andrea.mariscotti@unige.it

\section{INTRODUCTION}

The term "DC grid" may be considered as a catchall for various types of distribution networks that are being extensively used for a wide range of applications. One of the advantages of DC distribution is the ease of integration of sources and loads, without the complication of phase angle instability and coordination, typical of AC applications. Such networks are operated at both Medium Voltage (MV) and Low Voltage (LV) with some standardized nominal voltage values. Compared to AC grids in several cases DC grids can interface to pulse loads more effectively, that is with a faster response and without violating Power Quality (PQ) constraints [1]. The physical extension is variable, from some tens of meters within a large room, a smart house or an electrified vehicle, hundreds of metres between buildings, at a campus, or onboard ships, and up to some $\mathrm{km}$ for distribution within smart residential districts, technological parks, etc. [2], [3]. Some representative examples of such networks are as follows.

Smart data centres pursue a DC distribution perspective, in particular to ensure a high level of availability, using capacitors, batteries and autonomous renewable energy sources (photovoltaic and fuel cells to cite the most common ones operating at DC), covering a wide time scale of events (fluctuations, dips, short and long interruptions) [4].

MV power distribution onboard ships features various types of loads with different dynamics and power absorption levels [1],
[5], [6]. Various arrangements may be adopted: split DC and zonal bus types, different sub-bus solutions for generators, propulsion loads and PPLs, etc. [6]. The extension of the network is of course limited to the physical size of the ship (up to about $300 \mathrm{~m}$ ) but cabling and routing may be quite complex.

Another example of $\mathrm{LV} / \mathrm{MV}$ application characterized by dynamic moving loads is the wide set of railways, metros, tramways and trolley buses, all supplied from a catenary (or third rail) system fed by rectifier substations [7], [8]. The line voltage is generally standardized to a set of nominal values of 600,750 , 1500 and $3000 \mathrm{~V}$. These networks feature the largest extension, as they cover entire cities, regions and countries. They are sectioned, however, into smaller portions mainly for an exigency of maintenance and operation, besides control of supply voltage. The network frequency response is nevertheless peculiar, with significant variations of the network impedance and resonances already appearing at low frequency [9].

Loads with large nominal power and significant dynamics have a direct impact on the line voltage (subject to appreciable variations consequential to load cycles) and can compromise network stability [10]. As in all distribution networks, the primary quantity is the voltage available at equipment terminals, but for a complete assessment of the network-load interaction, current and power should be also evaluated. In particular, the current waveform is specifically involved in phenomena such as inrush, identification of faults and in general control of the interface converters by feed-forward methods, attempting dynamic 
impedance control. The power profile instead is the primary quantity for the power and energy budget, as well as for prediction and control [11].

In general, the impact of Pulsed Power Loads (PPLs) is reduced by a range of techniques suitable for DC grids, whose applicability may depend on the specific grid characteristics:

- first of all load dynamics may be limited at the origin, e.g. by limiting the rate of rise of the power demand, compatible with the load mission and characteristics; a wide range of techniques have been proposed, falling all under a unique category that we may call "profile-based control"

- additional passive energy storage devices (e.g. batteries, supercapacitors, etc.) may be installed, capable of feeding the supplementary power with the required rapidity, and at the same time dampen network swell (consequential to load release) and recover the energy, in case of regenerative behaviour

- $\quad$ the role of passive energy storage devices may be backed up by the so called "DC springs" and other active compensation devices.

The work is thus structured starting from PPL characteristics in terms of electrical behaviour and interaction with the DC grid (Section 2). Section 3 then focuses on metrics suitable to describe quantitatively such interaction and on the impact on DC grid operation and PQ. These metrics are derived from previously proposed PQ indexes, as well as from considerations regarding current and power absorption profiles. Section 4 summarizes the normative PQ requirements for DC grids in various applications that are characterized by the presence of PPLs, such as avionics, shipboard and railways at various extents. Section 5 then evaluates the performance of the proposed metrics, using simulated and measured data.

\section{PULSED POWER LOADS AND NETWORK INTERACTION}

Pulsed Power Loads (PPLs) with repeated large variations of load current represent an issue to DC grid voltage stability and regulation [12]. For complex systems with non-essential and essential loads, the adoption of a DC zonal distribution system is commonplace, dividing the DC grid onboard into zones separated by interface converters. Each sub-grid adopts specific solutions where necessary for critical loads, de facto confining and limiting the propagation of transients and disturbance. Since power is fed often by intrinsically AC sources (such as Gas Turbine and Diesel Engine alternators), heavy loads may be instead fed directly from a primary AC grid, from which the other zoned DC sub-grids are derived, one or more dedicated to specific PPL and local energy storage.

PPL loads are usually interfaced with a DC/DC front-end converter, based on a variety of topologies, depending also on power rating, voltage level and required dynamics. PPLs for a wide range of applications may be exemplified as follows.

- Devices and apparatus that are part of scientific experiments in nuclear physics, such as the magnetic circuits of acceleration and bending at synchrotrons, the Large Hadron Collider, etc. In several cases choices are a compromise between desirable performance for new types of experiments reutilization of existing equipment (undergoing thus gradual modernization) and exigencies of power absorption, feasibility of protections, and continuity of service. DC distribution facilitates the direct or indirect connection of energy storage, ensuring both fast dynamic response and higher continuity of service, even supporting reconfiguration on the fly ("hot swap") to account for some amount of DC/DC supply going out of service [13].

- Radar systems connected to aircraft DC grid with power absorption following transmission pulses. The DC grid onboard aircrafts is characterized by a limited power deployment, with flight control loads, constant power loads (such as fuel pump), and the said radar load, as well as modern radios. The load profile described in [14] may be taken as an exemplifying case: current pulses of $4 \mathrm{~ms}$ duration and peak power of $33.6 \mathrm{~kW}$ with respect to a steady power absorption of one third $(11.2 \mathrm{~kW})$; peaks are arranged in pulse bursts with spacing of $10 \mathrm{~ms}$ ( 5 peaks) with a repetition of $200 \mathrm{~ms}$. Modern aircrafts may be equipped with more than one radar device, for which coordination for interleaved operation may be a strategic option. Modern radio systems with advanced characteristics of spectrum exploitation, long range and traffic acceptance flexibility (especially for military applications) may share a power absorption pattern similar to radars.

- Rail guns and electromagnetic weapons, such as high-power laser and microwave beams, typically located onboard ships [15]. All these weapon systems deliver peak power levels in the order of $1 \mathrm{GW}$ or more through a pulse forming network, whose charging from the ship DC grid absorbs power levels in the order of $10 \mathrm{MW}-30 \mathrm{MW}$ for a duration of some seconds. During intensive use DC grid loading is almost continuous, with the delivery of fast energy pulses that may easily decoupled thanks to the much higher characteristic frequency.

- Electrified transports featuring high-performance units with a dynamic power profile. Two quite different modern transportation means fall into this category: urban highspeed high-pace electrified guideway systems and electric vehicles with dynamic wireless power charging, with pulsed power absorption with fast charging times when passing charging points. Both can be assumed fed by a DC grid although the physical extension is quite different: dynamic wireless power transfer requires local DC distribution buses up to about some hundreds meters [16], whereas guideway systems, tramlines and railways of various kids feature supply sections of several $\mathrm{km}$ [17]. For guideway systems, such as metros, it must be pointed out that PPLs at the load release or when implementing regenerative braking, may cause a significant increase of line voltage with consequence for electrical safety, especially for passengers and people standing at platform and near platform screen doors [18].

\section{DISTURBANCE METRICS}

For both the implementation of the control policies and the assessment of performance, suitable metrics of the disturbance are necessary. Such metrics can be applied to voltage, current, power, or a combination thereof (multi-criteria metrics). They were preliminarily discussed and compared to relevant standards in the IMEKO TC4 conference paper [19], of which this work is the continuation, with focus on dynamic PPL loads and network stability.

From the normative viewpoint [20]-[25] transient electrical phenomena may be classified as:

- macroscopic voltage variations lasting for tens ms or longer

- $\quad$ shorter voltage variations, related to switching transients of variable amplitude 
- periodic variations often described in terms of harmonic distortion or ripple, covering a wider frequency range.

It is commonplace to evaluate all these phenomena by the instantaneous difference with respect to steady state $V_{\mathrm{dc}}$ value [1], [12], applying the concept of ripple to all voltage variations, both transient and repetitive. The MIL-STD-1399-300 standard [26] has recently extended the concept of distortion and ripple to the active power absorption profile itself for AC grids onboard US Navy ships. The concept is interesting for DC grids where the concept of absorbed power is more straightforward and does not involve reactive power.

In the following, voltage fluctuations and transient variations are considered first, discussing alternative metrics other than ripple; periodic variations and ripple are then considered, including the extension to cover aperiodic variations and transients.

\subsection{Voltage fluctuation}

As anticipated the network response and the quality of the delivered energy (Power Quality in a wide perspective) is usually measured by indexes applied to the line voltage, measuring voltage spread during fluctuations to compare with maximum values ad time-amplitude limit curves.

Line transients may thus be evaluated by a measure of their pure amplitude $A$ (e.g. peak or average value), equivalent time duration $T_{X}$ (e.g. half-amplitude duration $T_{50}$ ) and combined amplitude-duration. The first index that has a strong relationship with AC networks is the rms value, that when defined for aperiodic phenomena corresponds in reality to a measure of the area of the signal. Thus, focusing on aperiodic phenomena for generality, the combination of amplitude and duration gives two measures of the intensity of the phenomenon: area and energy. Taking a time interval $\left[t_{1}, t_{2}\right]$ and considering the AC portion $x(t)$ of the network quantity (voltage or current), having first subtracted an estimate of the steady value $X$, we obtain:

$$
S=\int_{t_{1}}^{t_{2}} x(t) d t, \quad E=\int_{t_{1}}^{t_{2}}|x(t)|^{2} d t, \quad P=\frac{E}{t_{2}-t_{1}} .
$$

Energy is calculated and considered in a signal processing perspective, as the square of a signal $x$ over the time interval $\left[t_{1}, t_{2}\right]$. Then power $\mathrm{P}$ is just obtained as energy $\mathrm{E}$ divided by the duration $t_{2}-t_{1}$ of the time interval. It is easy to see that combining voltage and current corresponds to the squared rms.

The mean square time duration may be also defined, that measures the interval where the energy is concentrated:

$$
\tau^{2}=\int_{t_{1}}^{t_{2}} t^{2}|x(t)|^{2} \mathrm{~d} t / \int_{t_{1}}^{t_{2}}|x(t)|^{2} \mathrm{~d} t .
$$

Area (or impulse strength) and energy can be used to evaluate the impact on loads: the missing area or energy of a negative transient (a temporary reduction of the line voltage) will cause a reduction of the relevant quantities at the load side. The consequence is a diminished performance that may vary for duration and severity depending on the load type. Such impact is partially compensated by filters (capacitors mainly), storage devices (e.g. batteries and supercapacitors) and DC springs over different time scales, covering fast and slow variations. In addition, control at the source with a reserve of energy driven by a suitable index of the network quality of power can support cooperative control.

\subsection{Voltage ripple}

DC grids are generally characterized by means of "ripple", that EN 61000-4-17 [27] describes as composed of "power frequency or its multiple 2, 3 or 6", focusing with a limited view on the mechanism of production by classic AC/DC conversion (based e.g. on diode and thyristors rectifiers). This scheme is well represented in DC electrified transports where the ripple of substation rectifiers is clearly visible: its relevance is in terms of line voltage fluctuation reflected also in the track voltage, where a significant amount of ripple may be relevant for the assessment of touch voltage and body current [18]. Ripple is thus a repetitive phenomenon superimposed to the DC steady value. Modern $\mathrm{AC} / \mathrm{DC}$ and DC/DC converters and poly-phase machines for renewable energy sources are used extensively to improve PQ and for ease of interfacing in modern micro-grids and smartgrids. This leads necessarily to a reformulation of the concept of ripple to a more general definition accounting for nonharmonically related components, possibly non-stationary.

The time domain definition of ripple is the maximum instantaneous voltage variation over a given time interval [28][29] or directly as difference between the observed maximum and the minimum voltage values [24], both weighted by the steady dc voltage value $V_{\mathrm{dc}}$. In this perspective ripple and voltage fluctuation are synonymous, provided that ripple is defined as difference with respect to $V_{\mathrm{dc}}$, taking thus positive and negative values (above or below $V_{\text {dc }}$ ) separately, allowing to consider asymmetric limits for voltage variation.

It is observed that DC networks have a much lower harmonic content thanks to the large deployed capacitance and in general the lower impedance in the harmonic frequency range, as it was demonstrated for railways in [30], comparing harmonic power terms in AC and DC systems. Lower network impedance keeps harmonic voltage components low, while amplifying current distortion at higher frequency, as sources see a quasi-short-circuit condition.

Basically speaking, ripple may be calculated as the maximum of the peak-to-peak or peak excursion of network voltage [12], [28], but other measures of it (rms, percentiles, ...) were proposed in the past [31]-[33]. Ripple addresses two objectives at once, quantifying the spread of instantaneous values and the network distortion (ripple was defined both in time and frequency domain [28], [29]). The explicit connection between ripple and DFT (including harmonics as such and other components) was given in [28] with the index $D_{L F S D}$ and in [31] with RDF.

Ripple can describe the quality of the delivered voltage in terms of fluctuations and excursion, as well as assessing significant load steps and inrush phenomena when applied to the current waveform.

\subsection{Power trajectory}

PPL relevance is caused by the sudden power absorption and the line voltage drop consequential to the flow of absorbed current. Grid voltage will change by tens of $\%$ when current increases by orders of magnitude, so that at a first approximation metrics based on power may be replicated for current with similar results.

PPL input power as a function of time $P_{P}(t)$ is commonly named "power trajectory". It has an approximately trapezoidal shape with amplitude $P$, comparable rise and fall time $t_{r}$ and $t_{f}$, and top value duration $t_{p}$; pulses of overall duration $T_{p}$ repeat in bursts of duration $T_{b}$, that may occur more than once forming a train of bursts, one burst every $T_{t}$ seconds, as sketched in Figure 
1. The radar load depicted in Section 2 fits this description with $t_{p}=4 \mathrm{~ms}, T_{p}=10 \mathrm{~ms}, T_{b}=50 \mathrm{~ms}$ and $T_{t}=200 \mathrm{~ms}$.

For our purpose the following elements are particularly important and characterize the power trajectory: the top value $P$, the rate of rise $P / t_{r}$, the duration of the power pulse $T_{P}$ and the repetition intervals, $T_{b}$ and $T_{t}$.

The peak power $P$ establishes the loading of the source and the grid voltage reduction, taking one pulse alone in almost steady conditions.

The rate of rise instead indicates the dynamics of the power demand and the rapidity with which the DC grid and its energy storage must feed the load to sustain the network voltage. A metric that weights the rate of rise is the derivative vs. time of the power trajectory.

The pulse duration $t_{p}$, together with the absorbed power $P$, is relevant from an energy viewpoint, indicating the level of depletion of the energy storage during one power absorption event. The two parameters may be combined by calculating the area $A_{P}=P t_{p}$ of the power trajectory pulse, that as a general metric was introduced in section 3.1.

$T_{b}$ and $T_{t}$ indicate instead the periodicity and the amount of time available to recharge the energy storage devices between two pulses or two bursts of pulses. The number of pulses in one burst and of bursts in one train come directly as $N_{p}=T_{b} / T_{p}$ and $N_{b}=T_{t} / T_{b}$. Combined with the area $A_{P}$, that has the unit of measure of energy, we obtain the total depletion in terms of number of pulses, and the rate of depletion of the energy storage, that must be matched by the source capability.

The time rate of change of the power trajectory was proposed as the reference quantity of a disturbance metric to apply to PPLs [11]:

$$
d_{P}=\sqrt{\frac{1}{T_{P}} \int_{0}^{T_{P}}\left(\frac{\mathrm{d} P_{P}(t)}{\mathrm{d} t}\right)^{2} \mathrm{~d} t}
$$

The expression for $d_{P}$ returns the average square derivative of the power trajectory $P_{P}(t)$; in other words, this is the rms value of the derivative of the power trajectory. Considering the sinusoidal components composing it, the time derivative corresponds to a multiplication by $\omega$ of each component; the structure of this penalization is similar then to the one adopted for high-voltage stress of capacitor insulation [34] (the reference quantity being there the electric field or the terminal voltage).

By considering the power trajectory expressed as $P_{P}(t)=v(t) \times i(t)$, the derivative and square of the product may be further developed. The derivative of a product is equal to the sum of the two mixed products of one of the quantities with the

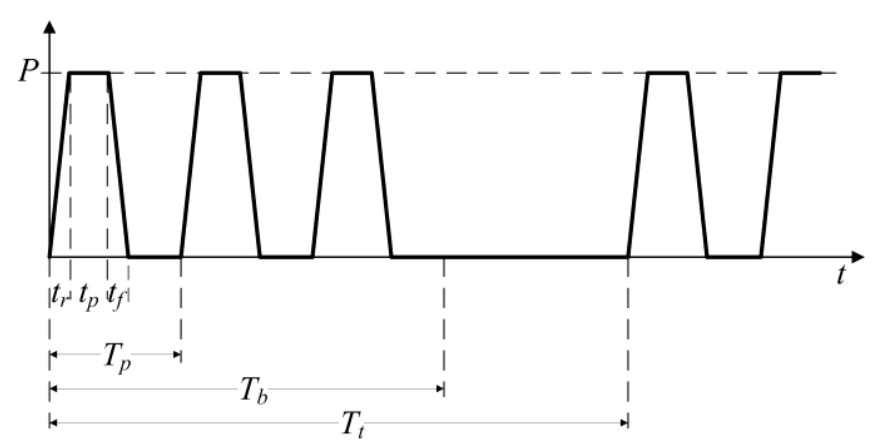

Figure 1. Ideal power trajectory $P_{P}(t)$ of a PPL, absorbing power in bursts of individual approximately trapezoidal pulses. derivative of the other. Each derivative is the multiplication by $\omega$ of the quantity expressed as a Fourier series:

$$
d_{P}^{\prime}=\sqrt{\frac{1}{T_{P}} \int_{0}^{T_{P}}\left(\sum_{m} \tilde{V}_{m}(t) \sum_{n} \omega_{n} \tilde{I}_{n}(t)+\sum_{m} \omega_{m} \tilde{V}_{m}(t) \sum_{n} \tilde{I}_{n}(t)\right)^{2}}
$$

having identified for brevity with $\tilde{V}_{m}(t)$ and $\tilde{I}_{m}(t)$ the Fourier series terms each with an exponential term and the peak value of the voltage or current component.

It is also observed that to the aim of calculating energy, only the terms with a non-null integral over the longest period are retained, that is the mixed products of $\tilde{V}_{m}(t)$ and $\tilde{I}_{n}(t), m \neq n$, give a null contribution and only those with the same harmonic index are retained. The peak values in $\tilde{V}_{m}(t)$ and $\tilde{I}_{n}(t)$, once passed through the integral over $T_{P}$, are transformed into rms values $V_{m}$ ad $I_{n}$ with a scaling of $1 / \sqrt{ } 2$ each that compensates with the factor of 2 resulting from the sum left and right of identical terms.

$$
d_{P}^{\prime}=\sqrt{\left(2 \sum_{n} \omega_{n} \frac{1}{2} V_{n} I_{n}\right)^{2}}=\sum_{n} \omega_{n} V_{n} I_{n}=\sum_{n} \omega_{n} P_{n} .
$$

The resulting alternative power rate metric $d_{P}^{\prime}$ is thus a harmonic active power term multiplied by the pulsation, representing a sort of weighting function. Harmonic active power was analysed for DC and AC railway systems in [30], finding that relevant terms are located at low frequency with relevance of a fraction of $\%$ compared to the total exchanged energy. Such terms are larger for AC than for DC systems, that feature in general larger deployed capacitance (and may be also backed up by various energy storage systems).

\subsection{Power distortion}

Treating the absorbed power profile (i.e. the power trajectory $\left.P_{P}(t)\right)$ as a signal, the equivalent of the Total Harmonic Distortion may be calculated, as well the intensity of spectral components of $P_{P}(t)$ for specific frequency intervals. This is the approach recently proposed in the MIL-STD-1399-300 interface standard for AC grids onboard US Navy electric ships. Power Distortion (PD) is calculated for the active power component only (called "real power"):

$$
P D=\sqrt{\sum_{n}\left(P_{n} / \sqrt{2} P_{a v}\right)^{2}} .
$$

Such a metric speaks of peak value of active power at a given frequency interval (or bin $n$ ) and extracts its rms value by dividing by $\sqrt{ } 2$. This metric is preliminarily considered here for DC systems by simply analysing the spectral behaviour of $P_{P}(t)$, compared to the expression derived for $d_{P}^{\prime}$ in (4), where the active power terms are arithmetically summed with the inclusion of pulsation as relative weight. Considering the typical behaviour and response of the elements of a DC grid (energy storage devices, filters, converter controls), we briefly observe that an increased weight linear with frequency does not express a practical scenario where high frequency is in reality filtered by capacitors, whereas medium frequency components may fall beyond control bandwidth. 


\section{NORMATIVE REQUIREMENTS}

\subsection{Voltage variations, swells and sags (dips), and interruptions}

Overvoltages and undervoltages may be named swells and sags (or dips) with analogy to AC networks. Standards specifying requirements for $\mathrm{PQ}$, performance and reliability of DC distribution divide among the areas of application: MIL-STD704F [23] for aircrafts; IACS Reg. E5 [22] and IEEE Std. 45.1 [21] for ships; EN 50155 [24] onboard rolling stock; ITU-T L.1200 [25] for telecommunication and data centers.

In general, distinction is needed from other long-term transients (long interruptions and fluctuations), as well as from very short-term phenomena, usually classified as spikes or surges. These latter in DC grids with no overhead lines are in general of modest amplitude, thanks to the large deployed capacitance (batteries and other energy storage devices, output filters of interfacing converters, etc.) and the consequent low transient impedance).

Definitions of IEEE Std. 1159 [35] and EN 61000-4-30 [36] for AC systems indicate a voltage dip or swell when the rms crosses a threshold, its duration quantified measuring the time interval between two consecutive crossings [35][37]. This definition based on the rms value is possible because swells and dips are defined for durations $>1$ cycle. The analogy with DC systems is not perfect and various time scales for the estimate of the transient and related steady value may be used. This will be shown with an example in Section 5.

Table 1 shows limits and reference values for voltage fluctuations, swells, sags and interruptions taken from the most relevant standards for the environment of application of PPLs reviewed in Section 2.

Figure 2 gives insight in graphical form into the timeamplitude limits of MIL-STD-704F and EN 50155 for aircraft and railway applications, respectively.

Faster transients (with shorter duration and rise and fall times) are more application specific and may depend e.g. on switching operations. A peculiar source of repetitive fast transients in

Table 1. Limits and reference values for transient events (voltage variation, dip and interruptions) ( $E=$ emission, I=immunity, $A=$ ambient specification).

\begin{tabular}{|c|c|c|c|c|}
\hline Standard & Phenomenon & Type & $\begin{array}{l}\text { Nom. } \\
\text { Volt. } U_{n} \text { in V }\end{array}$ & Ref. values \\
\hline MIL-STD-704F & Voltage var. & A & $28 \mathrm{~V}, 270$ & see Figure 1 \\
\hline EN 61000-4-29 & Voltage var. & 1 & $24-110$ & $\begin{array}{c}85-120 \% \\
0.1-10 \mathrm{~s}\end{array}$ \\
\hline EN 61000-4-29 & Voltage dip & 1 & $24-110$ & $\begin{array}{l}40,70 \% \\
0.01-1 \mathrm{~s}\end{array}$ \\
\hline EN 61000-4-29 & $\begin{array}{l}\text { Voltage interr. } \\
\text { HiZ \& LoZ }\end{array}$ & 1 & $24-110$ & $\begin{array}{c}0 \% \\
0.001-1 \mathrm{~s}\end{array}$ \\
\hline IACS & Voltage var. & 1 & $<1 \mathrm{kV}$ & $95-105 \%$ \\
\hline EN 50155 & Voltage var. & A & $24-110$ & see Figure 1 \\
\hline EN 50155 & Voltage interr. & A & $24-110$ & $\begin{array}{c}0 \% \\
0.01-0.03 \mathrm{~s}\end{array}$ \\
\hline L.1200 & Voltage var. & A & 300,380 & $\begin{array}{c}U_{n}-400-U_{n} 1 \mathrm{~min} \\
U_{n}-260-U_{n} 1 \mathrm{~min} \\
U_{n}-410-U_{n} 1 \mathrm{~s} \\
U_{n}-420-U_{n} 10 \mathrm{~ms}\end{array}$ \\
\hline L.1200 & Voltage dip & 1 & 300,380 & $\begin{array}{c}40 \% \\
0.01 \mathrm{~s}\end{array}$ \\
\hline L.1200 & Voltage interr. & 1 & 300,380 & $\begin{array}{c}0 \% \\
0.01 \mathrm{~s}(\mathrm{LoZ}) \\
1 \mathrm{~s}(\mathrm{HiZ})\end{array}$ \\
\hline
\end{tabular}

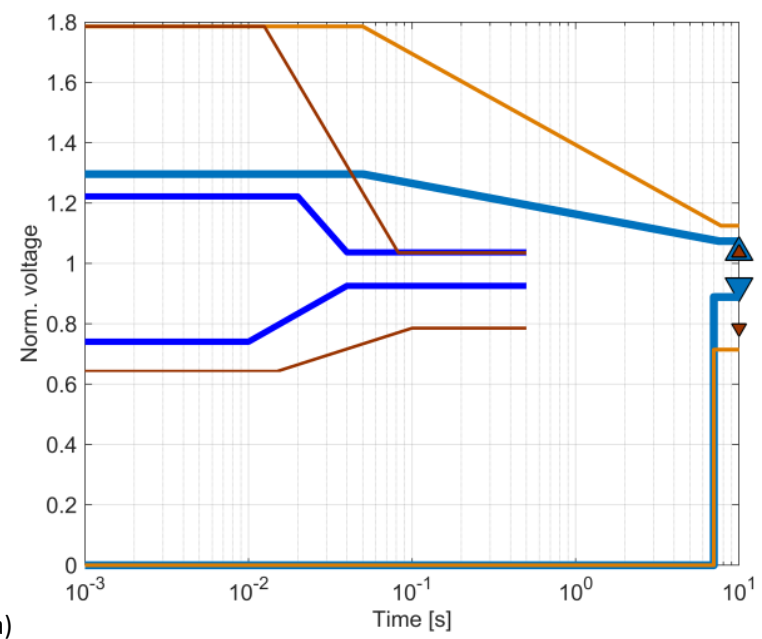

a)

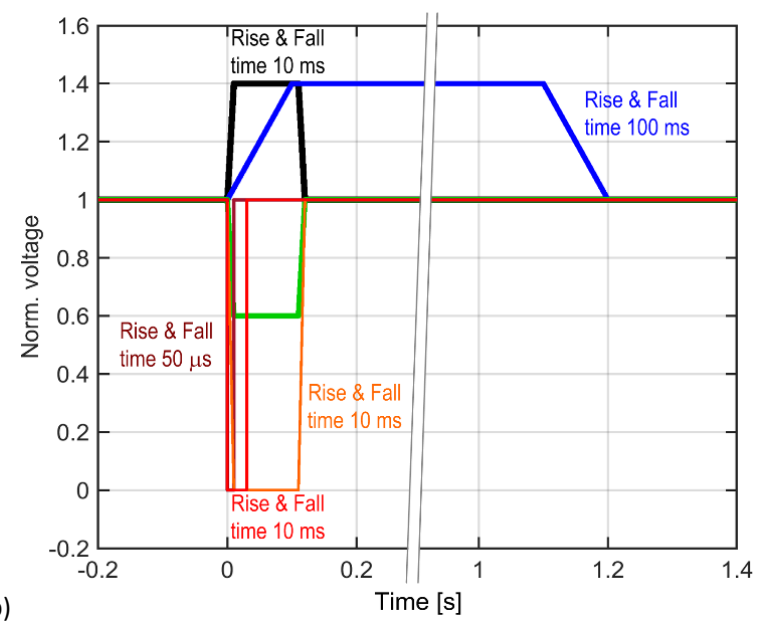

Figure 2. Profile of allowed transient overvoltage / undervoltage for (a) avionic (MIL-STD-704F) and (b) railway (EN 50155) applications.

electrified transportation systems are electric arcs, the known byproduct of the current collection mechanism, occurring at small discontinuities of the catenary line, during pantograph detachment in dynamic conditions and with adverse weather (e.g. ice) [38][39]. Such phenomenon is also relevant from an energy consumption viewpoint, in principle directly (as dissipative phenomenon in the arc itself and involved components), but most of all indirectly (interfering with the operation of the braking chopper of DC rolling stock) [40].

\subsection{Ripple, harmonics and periodic variations}

The EN 61000-4-7 [41] is a well-structured and complete standard that covers methods and algorithms quantifying spectral harmonic components, including interharmonics. However, the underlying assumption is always that of the existence of a fundamental, even for interharmonics, that e.g. combine mains and motor frequency in variable frequency drives or two mains frequencies in interface converters.

As observed in sec. 3.2, for DC grids it is always assumed that the DC supply comes from AC mains upstream, although technology advancement is characterized also by autonomous DC grids, supplied by sources that are intrinsically DC, such as fuel cells and photovoltaic panels. It is evident thus that concepts as distortion and spectrum components, and suitable limits, should be independent from a purely harmonic perspective:

- distortion becomes the amount of AC ripple superposed to the DC steady value $V_{\mathrm{dc}}$, rather than the composition of the 
amplitude of the harmonic components; this definition goes back to ripple, that candidates itself as a flexible and all comprehensive metric

- spectrum components are considered for a continuous frequency axis, although resolution frequency limitation is still relevant; this aspect is mentioned as MIL-STD-704F poses limits not only for distortion, but also to the spectral components [19].

Table 2 summarizes normative limits and reference values for ripple and distortion. Two quantities can be identified in the various standards that weight distortion and voltage variations:

- the distortion $D$ is the rms value of the ac components

- the distortion factor $D F=D / V_{\mathrm{dc}}$

- the ripple $R$ is the maximum absolute difference between an instantaneous value and the steady value $V_{\mathrm{dc}}$ [23]; an alternative definition is the difference of maximum and minimum of the line voltage divided by the half sum [24]; then, in some cases ripple is expressed as an rms quantity, so that for clarity the properly said ripple $R$ is considered as a "peak quantity".

Distortion may evidently accompany PPLs that are interfaced through static converters, but the most relevant feature is their impulsive and possibly repetitive power absorption profile. This profile for the considered dynamics may give rise to voltage and current components that may fall under the spectrum limitation at low frequency. For aircrafts in fact the MIL-STD-704F establishes limits of distortion components starting at $10 \mathrm{~Hz}$, unrelated from a concept of harmonic of a fundamental component.

\section{RESULTS AND DISCUSSION}

Before going into the various cases, it is remembered that in general the voltage and current profiles characterizing loads and PPLs have exponential profiles at the rising and falling edges, simply due to the most common behaviour of controls and electric network, featuring a dominant pole or two damped complex poles. Shorter spikes may be present, but their origin is exogenous, as they are caused by lightning and fault induced phenomena.

\section{1. $P Q$ indexes for typical PPL waveforms}

The behaviour of the metrics for transients $\left(S, E\right.$ and $\left.\tau^{2}\right)$ and ripple index discussed in Section 3.1 and 3.2, respectively, is analysed with respect to two typical PPL transient waveforms.

Case 1, shown in Figure 3, includes a voltage reduction due to a significant current absorption and a fast transient (swell) following the release of the load that returns to a negligible

Table 2. Limits and reference values for ripple and distortion ( $E=e m i s s i o n$, I=immunity, $A=$ ambient specification).

\begin{tabular}{llcccc}
\hline \multicolumn{1}{c}{ Standard } & Phenomenon & Type & $\begin{array}{c}\text { Nom. } \\
\text { Volt. } \boldsymbol{U}_{\boldsymbol{n}} \text { in V }\end{array}$ & Quantity & $\begin{array}{c}\text { Ref. } \\
\text { values }\end{array}$ \\
\hline MIL-STD-704F & Distortion & $\mathrm{A}$ & 28 & $\mathrm{D}$ & $3.5 \%$ \\
MIL-STD-704F & Ripple & $\mathrm{A}$ & 28 & $\mathrm{R}$ & $1.5 \mathrm{~V}$ \\
MIL-STD-704F & Distortion & $\mathrm{A}$ & 270 & $\mathrm{D}$ & $1.5 \%$ \\
MIL-STD-704F & Ripple & $\mathrm{A}$ & 270 & $\mathrm{R}$ & $6 \mathrm{~V}$ \\
IACS UR E5 & Ripple & $\mathrm{I}$ & & Rrms & $10 \%$ \\
EN 50155 & Ripple & $\mathrm{A}$ & $24-110$ & $\mathrm{R}$ & $5 \%$ \\
EN 61000-4-17 & Ripple & $\mathrm{I}$ & $24-110$ & $\mathrm{R}$ & $1,2.5,5$ \\
& & & & & \\
\hline
\end{tabular}

current absorption. The transient response is stable and characterized by a first-order exponential. As evident, the voltage reduction is slow and characterized by local changes of slope, visible in the moving average profile superposed to the original waveform. As anticipated, a simple criterion based on a crossing threshold may face difficulties when the rate of change is very slow and the slope is not uniform. Accurate and extensive noise removal is thus a minimum requisite.

Case 2, shown in Figure 4, shows the voltage and current waveforms of a periodic PPL with the initial transient followed by the oscillatory response of the generator control and a variable amount of high-frequency ripple, that may be caused by the interface converters or may be the symptom of an incipient resonance.

This waveform is quite similar to that of electric arc phenomena recognized in DC electric railways [39][42], where electric arcs trigger an impulsive reduction or increase of the line voltage (depending if the traction converter is absorbing power in a traction condition or injecting power into the network during a regenerative braking). The waveform in fact, besides a first pulse, features the oscillation of the rolling stock onboard filter (usually between 10 and $20 \mathrm{~Hz}$ ). Substation ripple at 300 and 600 $\mathrm{Hz}$, besides a more or less intense component at $100 \mathrm{~Hz}$, is always present and appears as soon as the major transient response components have vanished.

For Case 1 the calculated values for the two portions of the waveform (voltage reduction with negative slope and subsequent swell) of the waveform, and altogether, are reported in Table 3. The swell, although not so rapid, has a larger $\tau$ than the five-time longer voltage reduction that precedes it.

Case 2 instead is evaluated for both the three indexes $S, E, \tau^{2}$, and for ripple with a band limited approach as well, in order to separate the observed oscillations, at lower and higher frequency.

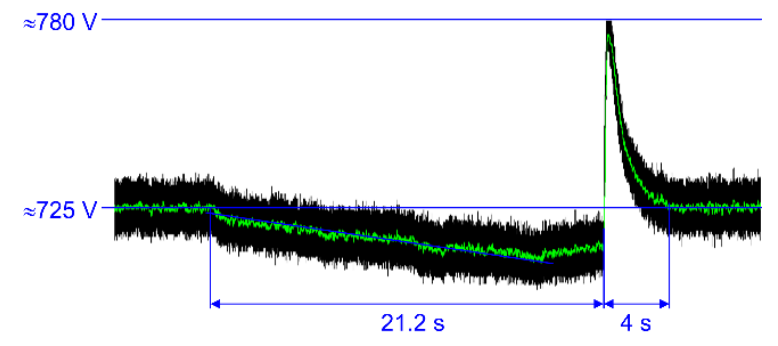

Figure 3. Network voltage following a significant current absorption by the PPL local storage with a sudden release at the end, causing an exponential transient increase (swell).

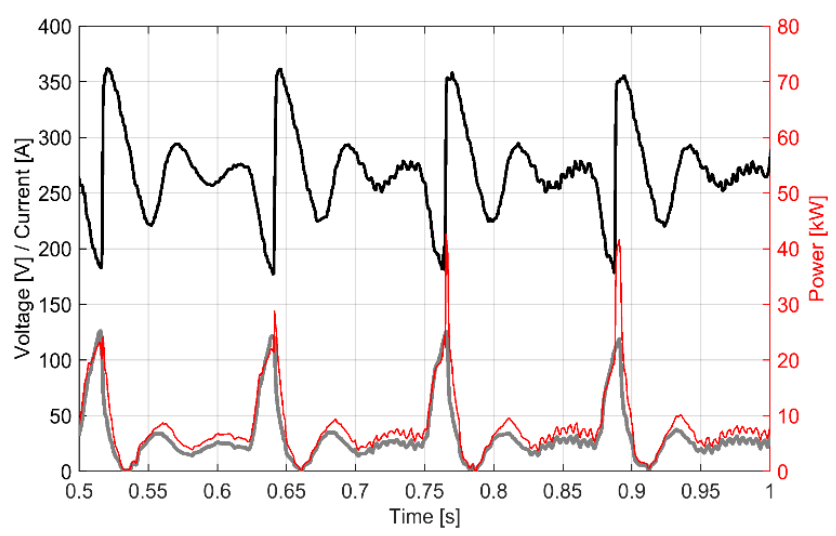

Figure 4. PPL profile with periodic pulsation and superposed ripple following the initial power absorption: voltage (black) and current (grey) use the left axis, power (red) uses the right axis. 
Since Case 2 shows four absorption pulses, index values are commented for equivalence between apparently similar events. The results are shown in Table 3, where the mean duration, and not its square, is reported for ease of comparison with signal duration and time intervals. The first voltage pulse has visibly less high-frequency oscillations, a slightly larger maximum value (the difference is about $3 \mathrm{~V}$, so less than $1 \%$ ), but no other apparent differences. Instead, the calculation of the energy points out an $8 \%$ larger value than the remaining pulses; analogously the mean duration is shorter by about $10 \%$, that means that the energy concentration is higher. The physical explanation may be a deeper charging of internal storage, that is then less depleted during the successive pulses. Indeed, the current peak in the first pulse is almost $4 \%$ higher.

Case 2 is then further analysed for what concerns ripple components, that visibly are located at two distinct frequency intervals, around about 25 and $200 \mathrm{~Hz}$, and whose intensity changes between periods of the pulsating waveforms of voltage and current of Figure 4. The band-limited ripple $R_{b p}[29]$ is thus calculated over different frequency intervals, using a windowed DFT (Hann window). From a preliminary evaluation of the band occupation, the frequency intervals should be separated at about $15 \mathrm{~Hz}$ and 50-100 Hz. With a sample frequency $f_{s}=2.048 \mathrm{kHz}$ (matched by applying resampling) the shown frequency resolution is $8 \mathrm{~Hz}$, to match the visible main oscillation preliminarily qualitatively estimated at $24-25 \mathrm{~Hz}$. Spectra shown in Figure 5 are cut at $400 \mathrm{~Hz}$. As briefly commented in the figure caption, the DFT of $P_{P}(t)$ highlights more effectively the spectrum pollution that may be a symptom of instability and that in a practical case should be further investigated.

\subsection{Spectrum of the PPL pattern}

The radar load profile [14], exemplified in section 2, is theoretically analysed for its frequency occupation, starting from the general PPL waveform of Figure 1. The characteristics of the waveform are summarized as: current pulses of $t_{p}=4 \mathrm{~ms}$ duration and peak power of $33.6 \mathrm{~kW}$ with respect to a steady power absorption of one third $(11.2 \mathrm{~kW})$; peaks are arranged in pulse bursts with spacing of $T_{b}=10 \mathrm{~ms}$ ( 5 peaks) with a train repetition interval of $T_{t}=200 \mathrm{~ms}$.

The coefficients of the Fourier series of a symmetrical trapezoidal pulse are reported in (6) [43]

$$
\begin{aligned}
c_{0} & =\frac{P}{p}, \quad q=\frac{T_{p}}{t_{r}}, \quad p=\frac{T_{p}}{t_{p}+t_{r}} \\
c_{k} & =\frac{2 P}{p} \cdot \frac{\sin (k \pi / q)}{k \pi / q} \cdot \frac{\sin (k \pi / p)}{k \pi / p},
\end{aligned}
$$

having defined $p$ and $q$ as the fraction of the half-value pulse duration $t_{p}+t_{r}$ and rise (or fall) time $t_{r}$ with respect to the duration of the single pulse $T_{p}$.

The resulting spectrum is a line spectrum with components repeating as per pulse spacing and modulated by the $c_{k}$ value. A

Table 3. Values of area $S$, energy $E$, and mean duration $\tau$ for the voltage waveforms of Case 1 and Case 2.

\begin{tabular}{cccc}
\hline Interval & $\boldsymbol{S}$ in V s & $\boldsymbol{E}$ in $\mathbf{V}^{\mathbf{2}}$ & $\boldsymbol{\tau}$ in $\mathbf{~}$ \\
\hline Case 1-intv 1 & 202.07 & 2200 & 8.80 \\
Case 1-intv 2 & 55.51 & 1725 & 10.25 \\
Case 2-intv 1 & 3.61 & 203.56 & 0.0308 \\
Case 2-intv 2 & 3.56 & 193.58 & 0.0343 \\
Case 2-intv 3 & 3.58 & 193.24 & 0.0353 \\
\hline
\end{tabular}

more complex expression may be derived without the simplifying assumption of equal rise and fall times. However, as evident from the curve shape of Figure 4, the trapezoidal curve shape is only an approximation and should not be assumed for more accurate assessment. The spectrum reported in Figure 5, instead, gives more reliable indication of the average behaviour of components over one $T_{p}$ interval. A more refined assessment of the peak power oscillation and its damping could be more effectively achieved by curve fitting in the time domain. In general, when the pulse duration is shorter and the oscillations are characterized by variable instantaneous frequency, the precautions and verifications discussed in [45] should be considered.

\subsection{Power rate metric $d_{P}$}

The power rate metric $d_{P}$ is evaluated with respect to a variable amount of harmonic distortion, using a realistic $P_{P}(t)$ trajectory with a pulsed profile. The first three periods of the red power curve of Figure 1, reconstructed from the measured voltage and current waveforms, are considered as cases of an increasing amount of distortion due to the visible superposed ripple. Such cases are indicated as Case 2-intv1, -intv2 and -intv3 for the first three pulse periods.

Synthetic results are shown in Table 4, reporting both the value of $d_{P}$ and the values of the previously defined indexes $S, E$ and $\tau$ for the selected intervals. It must be underlined that the two metrics $S$ and $E$ applied to $P_{P}(t)$ agree and weight more the
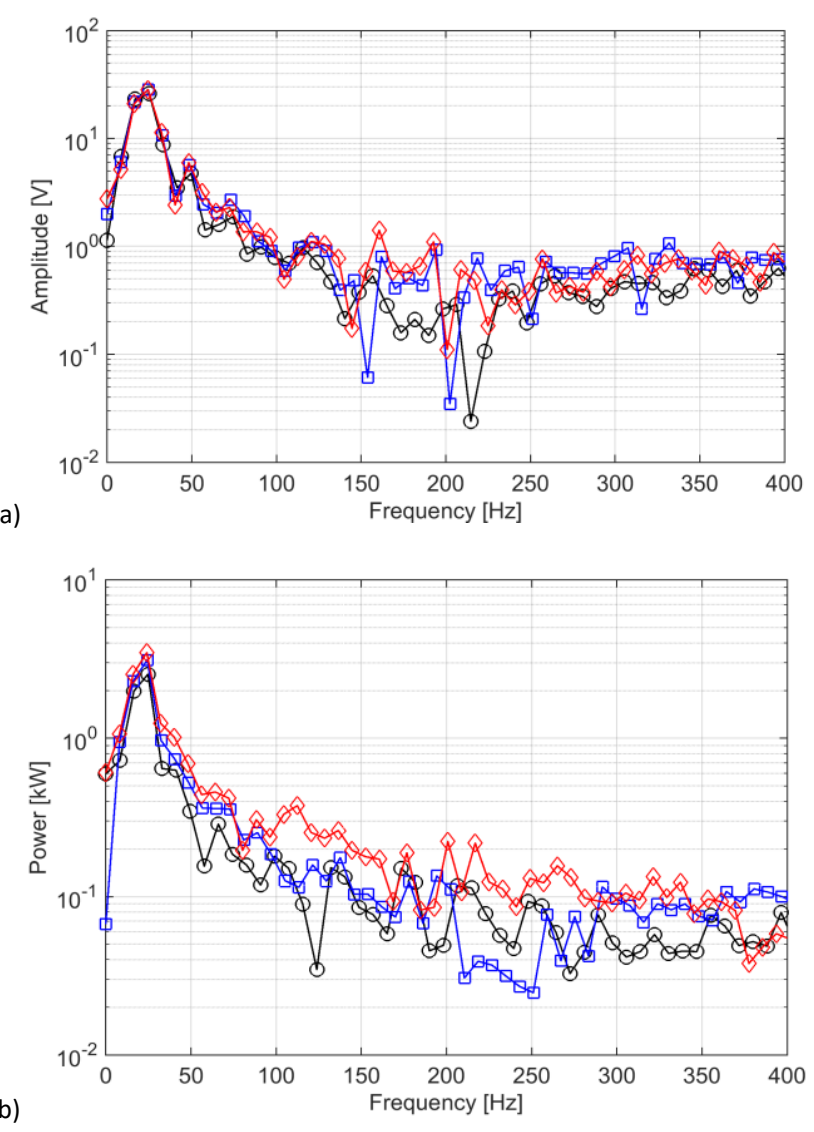

Figure 5. DFT of (a) $V(t)$ and (b) $P_{P}(t)$, for the three first pulses of Figure 4 (assigned colors black, blue and red). The main oscillation is clearly visible at about $24 \mathrm{~Hz}$, more stable in the $V(t)$ profile; the high-frequency ripple is more recognizable in the $P_{P}(t)$ spectrum as a general increase, rather than isolated and visible in the $250 \mathrm{~Hz}$ channel. 
Table 4. Values of metric $d_{P}$ compared to $S$ and $E$ for Case 2 applied to the power trajectory. $S$ and $E$ thus must be interpreted as "area" and "energy" of $P_{P}(t)$ considered as a signal and they do not have the same physical meaning The derivative operator is insensible to the steady $P_{P}(t)$ value, so that to calculate $S$ and $E, P_{P}(t)$ has been corrected by subtracting the steady value.

\begin{tabular}{ccccc}
\hline Case & $\boldsymbol{d}_{\boldsymbol{p}}$ diff in $\mathbf{W H z}$ & $\boldsymbol{d}_{\boldsymbol{p}}$ grad in $\mathbf{W H z}$ & $\boldsymbol{S}_{\mathbf{0}}$ in $\mathbf{W s}$ & $\boldsymbol{E}_{\mathbf{0}}$ in $\mathbf{W}^{\mathbf{2}}$ \\
\hline Case 2-intv 1 & $1.8410^{3}$ & $1.2810^{3}$ & 457.8 & $5.5510^{6}$ \\
Case 2-intv 2 & $1.5810^{3}$ & $1.2510^{3}$ & 470.2 & $5.6510^{6}$ \\
Case 2-intv 3 & $1.8610^{3}$ & $1.3910^{3}$ & 497.8 & $6.8910^{6}$ \\
\hline
\end{tabular}

larger peak value of the second and third pulse, but they ignore the increasing high-frequency ripple.

The $d_{P}$ metric is based on the derivative of the fed signal (the power trajectory) and has a significant variability depending on the implementation. Table 4 reports two versions of the calculation using "diff" (so the difference between adjacent vector components) and "gradient" (so the central difference between components with index differing by 2): the difference is significant especially because the two results do not have the same behaviour with respect to the interval number.

As a note, since the derivative operator is insensible to the steady value of the fed signal, $P_{P}(t)$ was fed to $S$ and $E$ also as an "ac signal", having subtracted the estimated steady value for each interval. For clarity $S$ and $E$ are thus indicated as $S_{0}$ and $E_{0}$.

\section{CONCLUSIONS}

This work has considered the presence of Pulsed Power Loads (PPLs) in DC grids with significant impact on network voltage variations and possibly on network stability. For the assessment of the impact and rating of various design solutions and implementations, suitable Power Quality metrics should be identified and applied. Such metrics have been discussed, focusing in particular on the quantification of network transients and accounting for the typical profile of absorbed power, named power trajectory.

These metrics may be divided in those weighting the characteristics of pulses and transients (area, energy, mean duration) and those dealing with characteristics that have been historically assigned to steady phenomena (ripple and distortion).

Metrics have been applied to voltage and current waveforms, but also to the power trajectory itself, as it has been recently proposed in the MIL-STD-1399-300 [26] for AC grids onboard US Navy ships. It is interesting to note how the power curve in one case of a pulsed waveform with increasing distortion anticipates and allows detection of this phenomenon more effectively than processing voltage or current waveforms alone.

The use and processing of power trajectory is particularly suited for PPLs and opens new possibilities of revisiting "traditional" PQ metrics with interesting performances and results. The better informative content of power with respect to voltage and current taken separately was pointed out in [46], when investigating internal and external sources of harmonic emissions in AC railways.

\section{REFERENCES}

[1] Y. Luo, S. Srivastava, M. Andrus, D. Cartes, Application of Disturbance Metrics for Reducing Impacts of Energy Storage Charging in an MVDC Based IPS, IEEE Electric Ship Technologies Symposium (ESTS), Arlington, VA, USA, 22-24 April 2013, pp. 287-291.

DOI: $\underline{10.1109 / E S T S .2013 .6523748}$
[2] S. Whaite, B. Grainger, A. Kwasinski, Power Quality in DC Power Distribution Systems and Microgrids, Energies, 8 (2015), pp. 4378-4399. DOI: $10.3390 /$ en 8054378

[3] V. A. Prabhala, B. P. Baddipadiga, P. Fajri, M. Ferdowsi, An Overview of Direct Current Distribution System Architectures and Benefits, Energies 11 (2018), no. 2463, 20 pp. DOI: $10.3390 /$ en11092463

[4] B. R. Shrestha, U. Tamrakar, T. M. Hansen, B. P. Bhattarai, S. James, R. Tonkoski, Efficiency and Reliability Analyses of AC and 380 V DC Distribution in Data Centers, IEEE Access 6 (2018), pp. 63305-63315.

DOI: $10.1109 /$ ACCESS.2018.2877354

[5] S. G. Jayasinghe, L. Meegahapola, N. Fernando, Z. Jin, J. M. Guerrero, Review of Ship Microgrids: System Architectures, Storage Technologies and Power Quality Aspects, Inventions 2 (2017), pp. 1-4.

DOI: $10.3390 /$ inventions2010004

[6] K. Kim, K. Park, G. Roh, K. Chun, DC-grid system for ships: a study of benefits and technical considerations, Journal of International Maritime Safety, Environmental Affairs, and Shipping, 2 (2018), pp. 1-12. DOI: $10.1080 / 25725084.2018 .1490239$

[7] X. J. Shen, Y. Zhang, S. Chen, Investigation of Grid-connected Photovoltaic Generation System Applied for Urban Rail Transit Energy-savings, IEEE Industry Applications Society Annual Meeting, Las Vegas, NV, USA, 7-11 October 2012, pp. 1-4. DOI: 10.1109/IAS.2012.6373995

[8] A. Hinz, M. Stieneker, R. W. De Doncker, Impact and opportunities of medium-voltage DC grids in urban railway systems, 18th European Conf. on Power Electronics and Applications (EPE Europe), Karlsruhe, Germany, 5-9 Sept. 2016, pp. 1-10.

DOI: $10.1109 /$ EPE.2016.7695410

[9] P. Ferrari, A. Mariscotti, P. Pozzobon, Reference curves of the pantograph impedance in DC railway systems, IEEE Intern. Conf. on Circ. and Sys., Geneve, Switzerland, 28-31 May 2000, pp. 555558.

DOI: $10.1109 /$ ISCAS.2000.857155

[10] P. Shamsi, B. Fahimi, Stability Assessment of a DC Distribution Network in a Hybrid Micro-Grid Application, IEEE Trans. Smart Grid 5 (2014), pp. 2527-2534. DOI: $10.1109 /$ TSG.2014.2302804

[11] J. M. Crider, S. D. Sudhoff, Reducing Impact of Pulsed Power Loads on Microgrid Power Systems, IEEE Trans. Smart Grid 1 (2010), pp. 270-277. DOI: $10.1109 /$ TSG.2010.2080329

[12] M. Steurer, M. Andrus, J. Langston, L. Qi, S. Suryanarayanan, S. Woodruff, P. F. Ribeiro, Investigating the Impact of Pulsed Power Charging Demands on Shipboard Power Quality, Proc. of IEEE Electric Ship Technologies Symposium, Arlington, VA, USA, 2123 May 2007, pp. 315-321. DOI: $10.1109 /$ ESTS.2007.372104

[13] European Synchrotron, EBS Storage Ring Technical Report, Sept. 2018 [Online]. Available: https://www.esrf.eu/files/live/sites/www/files/about/upgrade /documentation/Design\%20Report-reduced-jan19.pdf

[14] H. Ebrahimi, H. El-Kishky, M. Biswass, M. Robinson, Impact of Pulsed Power Loads on Advanced Aircraft Electric Power Systems with Hybrid APU, IEEE Intern. Power Modulator and High Voltage Conf. (IPMHVC), San Francisco, CA, USA, 6-9 July 2016, pp. 434-437.

DOI: $10.1109 /$ IPMHVC.2016.8012857

[15] J. J. A. van der Burgt, P. van Gelder, E. van Dijk, Pulsed power requirements for future naval ships, Proc. of 12th IEEE Intern. Pulsed Power Conf., Monterey, CA, USA, 27-30 June 1999, pp. 1357-1360 vol. 2. DOI: $10.1109 /$ PPC.1999.823779

[16] G. Guidi, S. D’Arco, J. A. Suul, A Modular and Distributed Grid Interface for Transformer-less Power Supply to Road-side Coil 
Sections of Dynamic Inductive Charging Systems, IEEE PELS Workshop on Emerging Technologies: Wireless Power Transfer, London, UK, 18-21 June 2019, pp. 318-323.

DOI: $10.1109 /$ WoW45936.2019.9030614

[17] S. Jung, H. Lee, C. S. Song, J.-H. Han, W.-K. Han, G. Jang, Optimal Operation Plan of the Online Electric Vehicle System Through Establishment of a DC Distribution System, IEEE Trans. Pow. Electron. 28 (2013), pp. 5878-5889.

DOI: $10.1109 /$ TPEL.2013.2251667

[18] A. Mariscotti, Electrical Safety and Stray Current Protection with Platform Screen Doors in DC Rapid Transit, IEEE Trans. Transp. Electrif., (2021) (in print).

DOI: $\underline{10.1109 / \text { TTE.2021.3051102 }}$

[19] A. Mariscotti, Overview of the Requisites to Define Steady and Transient Power Quality Indexes for DC Grids, Proc. of 24th IMEKO TC4 Intern. Symp., Palermo, Italy, 14-16 September 2020. Online [Accessed 20 June 2021]

https://www.imeko.org/publications/tc4-2020/IMEKO-TC42020-23.pdf

[20] G. Van den Broeck, J. Stuyts, J. Driesen, A critical review of power quality standards and definitions applied to DC microgrids, Applied Energy 229 (2018), pp. 281-288. DOI: $10.1016 /$ j.apenergy.2018.07.058

[21] IEEE Std. 45.1, IEEE Recommended Practice for Electrical Installations on Shipboard - Design, 2017.

[22] IACS, Electrical and Electronic Installations - E5: Voltage and frequency variations, 2019.

[23] MIL-STD-704F, Aircraft Electric Power Characteristics, w/Change 1, 2016.

[24] EN 50155, Railway applications - Rolling stock - Electronic equipment, 2017.

[25] ITU-T Std. L.1200, Direct current power feeding interface up to $400 \mathrm{~V}$ at the input to telecommunication and ICT equipment, 2012.

[26] MIL-STD-1399-300, Interface Standard Section 300, Part 1: Low Voltage Electric Power, Alternating Current, 2018.

[27] EN 61000-4-17, Electromagnetic compatibility - Part 4-17: Testing and measurement techniques - Ripple on d.c. input power port immunity test, 2009 .

[28] M. C. Magro, A. Mariscotti, P. Pinceti, Definition of Power Quality Indices for DC Low Voltage Distribution Networks, Proc. of IEEE Intern. Meas. Techn. Conf. IMTC, Sorrento, Italy, 20-23 April 2006, pp. 1885-1888. DOI: $10.1109 /$ IMTC.2006.328304

[29] A. Mariscotti, Methods for Ripple Index evaluation in DC Low Voltage Distribution Networks, IEEE Intern. Meas. Techn. Conf. IMTC, Warsaw, Poland, 2-4 May 2007, pp. 1-4.

DOI: $10.1109 /$ imtc. 2007.379205

[30] A. Mariscotti, Characterization of Active Power Flow at Harmonics for AC and DC Railway Vehicles, Proc. of IEEE Vehicle Power and Prop. Conf., Hanoi, Vietnam, 14-17 October 2019, pp. 1-7. DOI: 10.1109/VPPC46532.2019.8952310

[31] J. Barros, M. De Apráiz, R. I. Diego, Power Quality in DC Distribution Networks, Energies 12 (2019), no. 848, 13 pp. DOI: $10.3390 /$ en 12050848

[32] I. Ciornei, M. Albu, M. Sanduleac, L. Hadjidemetriou, E. Kyriakides, Analytical derivation of PQ indicators compatible with control strategies for DC microgrids, Proc. of IEEE PES PowerTech, 18-22 June 2017, Manchester, UK, pp. 1-6. DOI: $10.1109 /$ PTC. 2017.7981179

[33] A. Mariscotti, Discussion of Power Quality Metrics suitable for DC Power Distribution and Smart Grids, Proc. of $23^{\text {rd }}$ IMEKO TC4 Intern. Symp., Xi'an, China, 17-20 September 2017. Pp. 150-
154. Online [Accessed 20 June 2021]

https://www.imeko.org/publications/tc4-2019/IMEKO-TC4 2019-032.pdf

[34] G. C. Montanari, D. Fabiani, The effect of nonsinusoidal voltage on intrinsic aging of cable and capacitor insulating materials, IEEE Trans. Diel. Electr. Insul. 6 (1999), pp. 798-802. DOI: $10.1109 / 94.822018$

[35] IEEE Std. 1159, IEEE Recommended Practice for Monitoring Electric Power Quality, 2019.

[36] EN 61000-4-30, Electromagnetic compatibility - Part 4-30: Testing and measurement techniques - Power quality measurement methods, 2015.

[37] A. Florio, A. Mariscotti, M. Mazzucchelli, Voltage sag detection based on rectified voltage processing, IEEE Trans. Pow. Del. 19 (2004), pp. 1962-1967. DOI: $10.1109 /$ TPWRD. 2004.829924

[38] G. Crotti, D. Giordano, P. Roccato, A. Delle Femine, D. Gallo, C. Landi, M. Luiso, A. Mariscotti, Pantograph-to-OHL arc: conducted effects in DC railway supply system, Proc. of $9^{\text {th }}$ IEEE Intern. Workshop on Applied Meas. for Power Systems (AMPS), Bologna, Italy, 26-28 September 2018, pp. 1-6.

DOI: $10.1109 /$ AMPS.2018.8494897

[39] A. Mariscotti, D. Giordano, Experimental Characterization of Pantograph Arcs and Transient Conducted Phenomena in DC Railways, Acta IMEKO 9(2) (2020), pp. 10-17.

DOI: $10.21014 /$ acta imeko.v9i2.761

[40] A. Mariscotti, D. Giordano, A. Delle Femine, D. Gallo, D. Signorino, How Pantograph Electric Arcs affect Energy Efficiency in DC Railway Vehicles, IEEE Vehicle Power and Propulsion Conf., Gijon, Spain, 18 November -16 Decemer 2020, pp. 1-5.

DOI: $10.1109 /$ VPPC 49601.2020.9330954

[41] EN 61000-4-7, Electromagnetic compatibility - Part 4-7: Testing and measurement techniques - General guide on harmonics and interharmonics measurements and instrumentation, for power supply systems and equipment connected thereto, 2009.

[42] A. Mariscotti, D. Giordano, A. Delle Femine, D. Signorino, Filter Transients onboard DC Rolling Stock and Exploitation for the Estimate of the Line Impedance, Proc. of IEEE International Instrumentation and Measurement Technology Conference (I2MTC), Dubrovnik, Croatia, 25-28 May 2020. pp. 1-6. DOI: $10.1109 / \mathrm{I} 2 \mathrm{MTC} 43012.2020 .9128903$

[43] C. R. Paul, Bandwidth of Digital Waveforms, IEEE EMC Society Newsletter, no. 223, 2009, pp. 58-64. Online [Accessed 20 June 2021]

http://www.emcs.org/acstrial/newsletters/fall09/PracticalPaper s.pdf

[44] A. Pratt, P. Kumar, T. V. Aldridge, Evaluation of 400V DC distribution in telco and data centers to improve energy efficiency, 29th Intern. Telecommunications Energy Conf., Rome, Italy, 30 September 4 October 2007, pp. 32-39. DOI: $10.1109 /$ INTLEC.2007.4448733

[45] L. Sandrolini, A. Mariscotti, Impact of Short-Time Fourier Transform Parameters on the Accuracy of EMI Spectra Estimates in the $2-150 \mathrm{kHz}$ Supraharmonic Interval, Electr. Pow. Sys. Res. 195 (2021), 107130. DOI: $10.1016 /$ i.epsr.2021.107130

[46] A. Mariscotti, Experimental characterization of active and nonactive harmonic power flow of AC rolling stock and interaction with the supply network, IET Electr. Sys. Transp., (2021) (in print), pp. 109-120.

DOI: $10.1049 /$ els2.12009 